\title{
IS THERE A CRITICAL ACCELERATION FOR THE ONSET OF CONVECTION?
}

\author{
THORSTEN POESCHEL AND THOMAS SCHWAGER \\ Humboldt-Universität zu Berlin, Institut für Physik, \\ Invalidenstraße 110, D-10115 Berlin, Germany
}

\begin{abstract}
Suppose granular material is shaken vertically with $z(t)=$ $A_{0} \cos \left(\omega_{0} t\right)$. Can we expect to find convection if $A_{0} \omega_{0}^{2}<g$ ? By means of theoretical analysis and computer simulation we find that there is no critical $\Gamma=\left|A_{0}\right| \omega_{0}^{2} / g$ for the onset of convection. Instead we propose a modified criterion which coincides with $\Gamma=1$ for small frequency $\omega_{0}$.
\end{abstract}

\section{Introduction}

In vertically shaken granular material one observes various macroscopic effects, such as convection [1-3], surface fluidization [4-6], spontaneous heap formation [7, 8], surface patterns [9-12], oscillons [13] and others. The common feature of all these effects is that particles change their position with respect to each other. If a single hard particle is shaken, one agrees that it cannot move as long as during the entire period of shaking the acceleration due to gravity $g$ exceeds the upwards acceleration of the shaker. For the case of sinusoidal motion $z(t)=A_{0} \cos \left(\omega_{0} t\right)$ with amplitude this condition is given if $\Gamma=A_{0} \omega_{0}^{2} / g<1$.

We want to discuss the problem whether in a granular material particles at the surface can separate from each other even if $\Gamma<1$. At first glance the problem seems to be trivial, however, one has to take into account that there is a nonlinear interaction of touching grains.

There is a controversial discussion in the literature whether there is a critical value of Froude number $\Gamma_{c}=\left|A_{0}\right| \omega_{0}^{2} / g$ below which the above mentioned effects vanish. In many experimental observations (e.g. [1,5,8,

\footnotetext{
${ }^{0}$ In: H.J.Herrmann, J.-P. Hovi, and S.Luding (eds): "Physics of Dry Granular Materials", Kluwer (Dortrecht, 1998), p.625
} 
9, 14, 15]) and computer simulations (e.g. [15]) such a critical number $\Gamma_{c}$ was found. Several authors believe that the value is $\Gamma_{c}=1$. In numerical simulations, however, some authors have found surface fluidization and convection for $\Gamma \lesssim 1[3,6,16]$. Gallas, Herrmann and Sokołowski [17] concluded already 1992 from their numerical results "... that the current belief that $\Gamma$ determines the degree of fluidization is incorrect".

\section{A one dimensional continuum model}

We discuss the response of granular material with respect to vertical oscillation in the limit of a one dimensional approach: the lowest bead of a vertical column of $N$ identical spherical beads is shaken with $z_{0}=A_{0} \cos \omega_{0} t$ and the other beads move due to their interaction force and gravity $g$. Adjacent spheres interact with their next neighbors by the force

$$
F_{k, k+1}=-\sqrt{R}\left(\mu \xi_{k, k+1}^{3 / 2}+\alpha \dot{\xi}_{k, k+1} \sqrt{\xi_{k, k+1}}\right)
$$

with $\mu$ and $\alpha$ being elastic and dissipative material constants, i.e. functions of Young modulus, Poisson ratio and dissipation rate (for details see [18]). $\xi$ is the overlap $2 R-\left|z_{k}-z_{k+1}\right|$ of adjacent spheres of radius $R$ and mass $m$ at positions $z_{k}, z_{k+1}$. The height of the column is $L=2 N R$. The net force experienced by the $k$-th bead reads, therefore

$$
\begin{aligned}
& F_{k, k+1}-F_{k-1, k}=m \ddot{z}_{k}= \\
& \quad \sqrt{R} \mu\left[\xi_{k-1, k}^{3 / 2}-\xi_{k, k+1}^{3 / 2}\right]+\sqrt{R} \alpha\left[\dot{\xi}_{k-1, k} \sqrt{\xi_{k-1, k}}-\dot{\xi}_{k, k+1} \sqrt{\xi_{k, k+1}}\right](2)
\end{aligned}
$$

Introducing new coordinates $u_{k}=z_{k}-2 R k(k=0 \ldots N)$ the overlap of two adjacent spheres can be written as

$$
\begin{aligned}
\xi_{k, k+1} & =2 R-z_{k+1}+z_{k}=u_{k}-u_{k+1}=u(2 R k)-u(2 R(k+1)) \\
& =u(2 R k)-u(2 R k)-\left.2 R \frac{\partial u}{\partial z}\right|_{z=2 R k}=-2 R \frac{\partial}{\partial z} u_{k}
\end{aligned}
$$

In (3) the discrete value $u_{k}$ has been replaced by $u(2 R k)$ which allows for a Taylor expansion. Eq. (2) turns into

$$
\begin{aligned}
m \ddot{u}_{k} & =\frac{4}{3} \pi R^{3} \rho \ddot{u}_{k}=-\mu \sqrt{R}(2 R)^{\frac{3}{2}} A-\alpha \sqrt{R}(2 R)^{\frac{3}{2}} B \\
\ddot{u}_{k} & =-\frac{3}{\sqrt{2} \pi \rho R}(A \mu+B \alpha) \\
A & =\left(-\frac{\partial}{\partial z} u_{k}\right)^{\frac{3}{2}}-\left(-\frac{\partial}{\partial z} u_{k-1}\right)^{\frac{3}{2}} \\
B & =-\frac{\partial}{\partial z} \dot{u}_{k} \sqrt{-\frac{\partial}{\partial z} u_{k}}+\frac{\partial}{\partial z} \dot{u}_{k-1} \sqrt{-\frac{\partial}{\partial z} u_{k-1}}
\end{aligned}
$$


$A$ and $B$ can be evaluated by Taylor expansion

$$
\begin{aligned}
A & =\left(-\frac{\partial}{\partial z} u_{k}\right)^{\frac{3}{2}}-\left(-\frac{\partial}{\partial z} u_{k}+2 R \frac{\partial^{2}}{\partial z^{2}} u_{k}\right)^{\frac{3}{2}}=2 R \frac{\partial}{\partial z}\left(-\frac{\partial}{\partial z} u_{k}\right)^{\frac{3}{2}} \\
B & =-\frac{\partial}{\partial z} \dot{u}_{k} \sqrt{-\frac{\partial}{\partial z} u_{k}}-\left(-\frac{\partial}{\partial z} \dot{u}_{k}+2 R \frac{\partial^{2}}{\partial z^{2}} \dot{u}_{k}\right) \sqrt{-\frac{\partial}{\partial z} u_{k}+2 R \frac{\partial^{2}}{\partial z^{2}} u_{k}} \\
& \approx-2 R \frac{\partial}{\partial z} \dot{u}_{k} \frac{\partial}{\partial z} \sqrt{-\frac{\partial}{\partial z} u_{k}}-2 R \frac{\partial^{2}}{\partial z^{2}} \dot{u}_{k} \sqrt{-\frac{\partial}{\partial z} u_{k}} \\
& =-2 R \frac{\partial}{\partial z}\left(\frac{\partial}{\partial z} \dot{u}_{k} \sqrt{-\frac{\partial}{\partial z} u_{k}}\right)
\end{aligned}
$$

Eqs. (9) and (11) contain only local variables, therefore we can drop the index $k$ and the equation of motion (5) in continuum approximation including gravity reads

$$
\begin{aligned}
\ddot{u} & =-g-\frac{3 \sqrt{2}}{\pi \rho}\left\{\frac{\partial}{\partial z}\left[\mu\left(-\frac{\partial}{\partial z} u\right)^{\frac{3}{2}}+\alpha\left(-\frac{\partial}{\partial z} \dot{u} \sqrt{-\frac{\partial}{\partial z} u}\right)\right]\right\} \\
& =-g-\frac{\partial}{\partial z}\left[\kappa\left(-\frac{\partial}{\partial z} u\right)^{\frac{3}{2}}-\beta \frac{\partial^{2}}{\partial t \partial z} u \sqrt{-\frac{\partial u}{\partial z}}\right] .
\end{aligned}
$$

Eq. (13) defines the abbreviations $\kappa$ and $\beta$. We are interested in the critical parameters of driving $\left(A_{0}, \omega_{0}\right)$ when the $N$-th particle loses contact, i.e. when $u_{N}>u_{N-1}$. According to the nonlinear interaction of the particles the motion of all other spheres is not sinusoidal anymore, instead one finds a superposition of many frequencies. We define the "response" $R\left(\omega_{0}\right)$ as the ratio $A_{N} / A_{0}$ where $A_{N}$ is the amplitude of the $N$-th particle at frequency $\omega_{0}$ which can be calculated by convoluting the motion $z_{N}(t)$ with $\exp \left(i \omega_{0} t\right)$ and $A_{0}$ is the amplitude of the driving vibration. Suppose $A_{N} \omega_{N}^{2} / g \geq 1$ the $N$-th particle separates from the $N-1$-st. If we would find $A_{0}<A_{N}$ the critical Froude number $\Gamma_{c}=A_{0} \omega_{0}^{2} / g$ would be less than 1 . We will show that there is a range for $\omega_{0}$ where this is the case.

\section{Solution of (13) in the limit of frictionless motion}

The boundary condition for Eq. (13) is $(\partial u / \partial z)_{z=L}=0$. To find the strain of the material without external forcing (shaking) we consider the limit of no damping $(\alpha=0)$. With

$$
x=1-\frac{z}{L}, \quad \tau=\left(\frac{g \kappa^{2}}{L^{5}}\right)^{\frac{1}{6}} t, \quad \Omega=\left(\frac{L^{5}}{g \kappa^{2}}\right)^{\frac{1}{6}} \omega,
$$


Eq. (13) turns into

$$
\frac{\partial^{2} u}{\partial \tau^{2}}=-\gamma^{2}+\frac{1}{\gamma} \frac{\partial}{\partial x}\left[\left(\frac{\partial u}{\partial x}\right)^{\frac{3}{2}}\right] \text { with }\left(\frac{\partial u}{\partial x}\right)_{x=0}=0, \quad \gamma=\left(\frac{g^{2} L^{5}}{\kappa^{2}}\right)^{\frac{1}{6}}
$$

Eq. (15) is defined for $x \in[0,1]$, its time independent solution $U(x)$ is

$$
U(x)=\frac{3}{5} \gamma^{2}\left(x^{\frac{5}{3}}-1\right)
$$

The solution of (15) can be considered as a superposition of the static solution (16) and a perturbation $w(x, \tau)$. Inserting $u=U+w$ in (15) yields

$$
\begin{aligned}
\frac{\partial^{2} w}{\partial \tau^{2}} & =-\gamma^{2}+\frac{1}{\gamma} \frac{\partial}{\partial x}\left[\frac{\partial U}{\partial x}+\frac{\partial w}{\partial x}\right]^{\frac{3}{2}} \\
& =-\gamma^{2}+\frac{1}{\gamma} \frac{\partial}{\partial x}\left[\left(\frac{\partial U}{\partial x}\right)^{\frac{3}{2}}+\frac{3}{2} \sqrt{\frac{\partial U}{\partial x}} \frac{\partial w}{\partial x}\right] \\
& =\frac{3}{2} \frac{\partial}{\partial x}\left[x^{\frac{1}{3}} \frac{\partial w}{\partial x}\right]
\end{aligned}
$$

We are interested in standing wave solutions $w=T(\tau, \Omega) X(x, \Omega)$ of Eq. (17) and obtain

$$
\frac{\ddot{T}}{T}=\frac{3}{2 X} \frac{\partial}{\partial x}\left(x^{\frac{1}{3}} \frac{\partial X}{\partial x}\right)=-\Omega^{2}
$$

with $\Omega$ being a real number. Obviously for $T(\tau, \Omega)$ one gets $T \sim \exp (i \Omega \tau)$. The solution of the spatial equation

$$
\frac{3}{2} \frac{\partial}{\partial x}\left(x^{\frac{1}{3}} \frac{\partial X}{\partial x}\right)+\Omega^{2} X=0
$$

can be found using the Ansatz

$$
X(x, \Omega)=x^{\frac{1}{3}} f(y), \quad y=\frac{2}{5} \sqrt{6} \Omega x^{\frac{5}{6}}
$$

which yields

$$
y^{2} \frac{\partial^{2} f}{\partial y^{2}}+y \frac{\partial f}{\partial y}+\left(y^{2}-\frac{4}{25}\right) f=0
$$

Eq. (21) is the Bessel equation of order 2/5. Hence, the solution of (19) is

$$
X(x, \Omega)=\left(\frac{6}{25}\right)^{\frac{1}{5}} \Gamma\left(\frac{3}{5}\right) \Omega^{\frac{2}{5}} x^{\frac{1}{3}} J_{-\frac{2}{5}}\left(\frac{2}{5} \sqrt{6} \Omega x^{\frac{5}{6}}\right)
$$


An expression containing $J_{2 / 5}$ would be a solution too, however, it does not satisfy the boundary condition of (15). The factor has been chosen in order to assure $X(0, \Omega)=1$.

\section{The criterion for the onset of surface motion}

The above defined response $R$ is the ratio $A_{N} / A_{0}$ for given driving frequency $\omega_{0}$, or $\Omega_{0}$, respectively. Since the zeroth particle corresponds to $x=1$ and the $N$-th to $x=0$ we can write

$$
R^{-1}(\Omega)=\frac{X(1, \Omega)}{X(0, \Omega)}=X(1, \Omega)=\left(\frac{6}{25}\right)^{\frac{1}{5}} \Gamma\left(\frac{3}{5}\right) \Omega^{\frac{2}{5}} J_{-\frac{2}{5}}\left(\frac{2}{5} \sqrt{6} \Omega\right)
$$

The response $R$ is an amplification factor, therefore, the value $g / R(\Omega)$ is the critical acceleration of the driving vibration. $R$ is larger than 1 for all driving frequencies $\omega$. This means that for any driving frequency $\omega_{0}$ and driving amplitude $A_{0}$ the amplitude of the top particle of the column $A_{N}$ at frequency $\omega_{0}$ will be larger than $A_{0}$. Hence, for $A_{N} \omega_{0}^{2} / g=1$, i.e. when the $N$-th particle separates from the $N-1$-st, we find $A_{0} \omega_{0}^{2} / g=\Gamma_{c}<1$.

Therefore, we replace the condition $\Gamma \geq 1$ which was supposed to be the precondition for surface fluidization, convection etc., by

$$
\frac{A_{0} \omega_{0}^{2}}{g}=\Gamma \geq R^{-1}\left(\omega_{0}\right)
$$

The function $R^{-1}(\omega)$ over $\omega$ is drawn in Fig. 1 (full line). The curve reveals pronounced resonances at Eigenfrequencies $\omega_{k}$ where $R^{-1}$ becomes minimal. All experiments on surface fluidization and convection which can be found in literature have been performed far below the first resonance. Therefore, of particular interest to practical purposes is the limit of small frequency $\omega_{0}$, i.e. below the first Eigenvalue. The Taylor expansion of $R^{-1}(\Omega)$ for small $\Omega$ yields

$$
R^{-1}=1-\frac{2}{5} \Omega^{2}+\mathcal{O}\left(\Omega^{4}\right)=1-\frac{2}{5}\left(\frac{L^{5}}{g \kappa^{2}}\right)^{\frac{1}{3}} \omega^{2}+\mathcal{O}\left(\omega^{4}\right) .
$$

Given the container vibrates with frequency $\omega_{0}$. Then for the critical amplitude $A_{0}$ of the vibration when the top particle separates, i.e. when the material starts to fluidize one finds

$$
A_{0}=\frac{g}{\omega_{0}^{2}}-\frac{2}{5}\left(\frac{L^{5}}{g \kappa^{2}}\right)^{\frac{1}{3}}
$$

Surprisingly even for very small frequencies where $R^{-1} \rightarrow 1$ one finds that the critical amplitude is reduced by a constant as compared with $g / \omega_{0}^{2}$. 


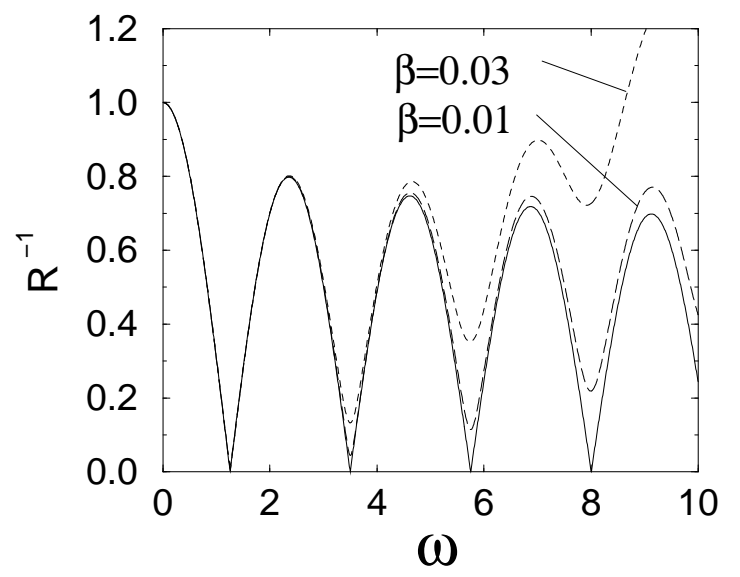

Figure 1. The reciprocal response $R^{-1}$ over driving frequency $\omega_{0}$ without damping, Eq. (23) (full line). The dashed lines show numerical results based on integration of Eq. (2) for damping $\beta=0.01$ and $\beta=0.03$.

\section{Numerical solution including damping}

Eq. (22) describes the behavior of the column of grains for the case of purely elastic contact $(\alpha=0)$. We have not been able to solve the full Eq. (13) including damping in closed form, therefore we have solved Eq. (2) numerically for different values of damping. The dashed lines in Fig. 1 display the reciprocal response $R^{-1}$ over $\Omega_{0}$ with fixed amplitude $A=$ $0.1 \mathrm{~mm}$, elastic constant $\kappa=2000 \mathrm{~m}^{7 / 2} / \mathrm{s}^{2}$ and $L=50 \mathrm{~m}$. Fig. 1 shows that for small frequency $\Omega_{0}$ and small damping $\alpha$ the theoretical curve (full line) agrees well with numerical data.

Hence, we believe that at least for small enough damping the results of the previous section remain correct, i.e. there is a region where one can find surface fluidization, convection and other macroscopic effects even if the maximum acceleration lies significantly below $g$.

\section{Conclusion}

We derived an equation of motion for a column of spheres on a vertically vibrating table, representing a granular material in one dimensional approximation. It is shown that the sphere on top of the column $N$ can separate from the $N-1$-st even if the table oscillates with $\left|A_{0}\right| \omega_{0}^{2} / g=\Gamma<1$. In agreement with numerical simulations by Gallas et al. [17] we came to the result that $\Gamma$ is not the correct criterion for classification of phenomena which occur in vibrated granular material.

We could show that instead of the widely accepted condition $A_{0} \omega_{0}^{2} / g>$ 1 one has to satisfy $A_{0} \omega_{0}^{2} / g>R^{-1}$ where $R^{-1}$ is a function of $\omega$ which is 
always less than one. Numerical calculations with low damping agree well with analytic results.

The described result is in contrast with several experimental investigations where a critical Froude number $\Gamma_{c} \geq 1$ has been measured. Whereas the Froude number is certainly the proper criterion to predict whether a single particle will jump on a vibrating table we suspect that this number is not suited to be a criterion for surface fluidization of a column of spheres, and the more not for a three dimensional granular material.

Acknowledgements. The authors wish to thank E. Clément, N. Gray, H. J. Herrmann, H. M. Jaeger, S. Luding, S. Roux and L. Schimansky-Geier for helpful discussions.

\section{References}

1. E. E. Ehrichs, H. M. Jaeger, G. S. Karczmar, J. B. Knight, V. Yu. Kuperman, and S. R. Nagel, Science, 267, 1632 (1995); J. B. Knight, E. E. Ehrichs, V. Yu. Kuperman, J. K. Flint, H. M. Jaeger, and S. R. Nagel, Phys. Rev. E, 54, 5726 (1996); J. B. Knight, H. M. Jaeger, and S. R. Nagel, Phys. Rev. Lett., 70, 3728 (1993).

2. J. A. C. Gallas, H. J. Herrmann, and S. Sokołowski, Phys. Rev. Lett, 69, 1371 (1992).

3. Y-h. Taguchi, Phys. Rev. Lett, 691367 (1992), Europhys. Lett., 24, 203 (1993).

4. S. Warr, J. M. Huntley, and G. T. H. Jacques, Phys. Rev. E, 52, 5583 (1995).

5. P. Evesque, E. Szmatula, and J. P. Denis, Europhys. Lett., 12, 623 (1990).

6. S. Luding, H. J. Herrmann, and A. Blumen, Phys. Rev. E, 50, 3100 (1994).

7. M. Faraday, Phil. Trans. R. Soc. Lond., 121, 299 (1831); F. Dinkelacker, A. Hübler, and E. Lüscher, Biol. Cybern., 56, 51 (1987); J. Rajchenbach, Europhys. Lett., 16, 149 (1991).

8. S. Douady, S. Fauve, and C. Laroche, Europhys. Lett., 8, 621 (1989); H. K. Pak and R. P. Behringer, Nature, 371, 231 (1994).

9. F. Melo, P. Umbanhowar, and H. L. Swinney, Phys. Rev. Lett., 72, 172 (1994).

10. T. Metcalfe, J. B. Knight, and H. M. Jaeger, Physica A, 236, 202 (1997).

11. K. M. Aoki and T. Akiyama, Phys. Rev. Lett., 77, 4166 (1996).

12. E. Clément, L. Vanel, J. Rajchenbach, and J. Duran, Jaques, Phys. Rev. E, 53, 2972 (1996).

13. P. B. Umbanhowar, F. Melo, and H. L. Swinney, Nature, 382, 793 (1996).

14. H. K. Pak, E. Van Doorn, and R. P. Behringer, Phys. Rev. Lett., 74, 4643 (1995); F. Melo, P. Umbanhowar, and H. L. Swinney, Phys. Rev. Lett., 75, 3838 (1995); P. Evesque and J. Rajchenbach, Phys. Rev. Lett., 62, 44 (1989); E. Clément, J. Duran, and J. Rajchenbach, Phys. Rev. Lett., 69, 1189 (1992); S. Fauve, S. Douady, and C. Laroche, J. Physique, C3, 187 (1989); P. Evesque, Cont. Phys., 33, 245 (1992); O. Zik and J. Stavans, Europhys. Lett., 16, 255 (1991).

15. J. J. Moreau, in: C. Thornton (ed.) Powders and Grains'93, p.227, Balkema (Rotterdam,1993).

16. G. C. Barker and A. Mehta, Nature, 364, 486 (1994); Phys. Rev. A, 45, 3435 (1992); Europhys. Lett., 27, 501 (1994); Y-h. Taguchi, Int. J. Mod. Phys. B, 7, 1839 (1993); T. Pöschel, and H. J. Herrmann, Europhys. Lett., 29, 123 (1995); J. A. C. Gallas, H. J. Herrmann, and S. Sokołowski, J. Physique. 2, 1389 (1992); L. Rolf, Lutz, ZementKalk-Gips, 46, 389 (1993).

17. J. A. C. Gallas, H. J. Herrmann, and S. Sokołowski, Physica A, 189, 437 (1992).

18. N. V. Brilliantov, F. Spahn, J. M. Hertzsch, and T. Pöschel, Phys. Rev. E, 53, 5382 (1996). 